\title{
Oxaliplatin-Induced Neurotoxic Side Effects and Their Impact on Daily Activities
}

Jenny Drott, Victoria Fomichov, Hans Starkhammar, Sussanne Börjeson, Karin I. Kjellgren and Carina Berterö

The self-archived postprint version of this journal article is available at Linköping University Institutional Repository (DiVA):

http://urn.kb.se/resolve?urn=urn:nbn:se:liu:diva-154883

N.B.: When citing this work, cite the original publication.

Drott, J., Fomichov, V., Starkhammar, H., Börjeson, S., Kjellgren, K. I., Berterö, C., (2019),

Oxaliplatin-Induced Neurotoxic Side Effects and Their Impact on Daily Activities, Cancer Nursing.

https://doi.org/10.1097/ncc.0000000000000674

Original publication available at:

https://doi.org/10.1097/ncc.0000000000000674

Copyright: Lippincott Williams \& Wilkins

Publisher URL Missing

Tweet 


\section{Abstract}

Background: Oxaliplatin (OXA) is frequently used in the treatment of patients with colorectal cancer (CRC) and OXA-induced neurotoxic side effects is common. Real time patient-reported neurotoxic side effects and impact on the patient's daily activities, are sparse in existing studies. Objective: This study aimed to identify and assess patient-reported OXA-induced neurotoxic side effects and their impact on the patient's daily activities, during and after chemotherapy. Methods: In a multicentre prospective longitudinal study, 46 chemo naïve patients with CRC treated with postoperative adjuvant OXA-based chemotherapy were monitored during treatment and at 3-, 6-, 9- and 12-month follow-ups. Patients were recruited from September 2013 to June 2016. In total, 370 OANQ (Oxaliplatin-Associated Neurotoxicity Questionnaire) responses were available for analysis. A mobile phone-based system was used to receive real time assessments. Results: All patients reported neurotoxic side effects and impact on daily activities during treatment. The side effects changed in character and body location over time, and had an impact on the daily activities.

Conclusions: The high prevalence of OXA-induced neurotoxic side effects significantly interfered with the patients' daily activities. We found significant differences between baseline data and follow-up time points for neurotoxicity, and the patients had not returned to baseline after one year.

\section{Implications for Practice:}

The real-time assessment using mobile phone technology seems to be a valuable tool for monitoring patient-reported neurotoxicity and interventions for tailored care. Effectively identifying neurotoxicity and its impact on the patient's daily activities is important in supportive cancer care. 
Key words: Cancer nursing, Chemotherapy, Colorectal cancer; Daily activities; mHealth; Neurotoxicity; Patient-reported outcomes, Symptom Control 
The platinum compound oxaliplatin (OXA) is a treatment option for patients with adjuvant treatment for resected colorectal cancer (CRC). Treatment related side effects as neurotoxicity is common and may impact the patient's everyday life during the cancer trajectory ${ }^{1,2}$. OXA has shown to improve survival and also disease control in the long term ${ }^{2,3}$. Neurotoxicity can interfere with the patient's daily living and affect ability to carry out activities. Because there is no evidence for effective treatment in terms of eliminating neurotoxic side effects, the most successful approaches include early identification, reduction of dose and interruption of treatment. Current research has identified a need for patient-oriented evidence in terms of longterm follow-up of neurotoxicity. However, neurotoxic side effects (NTS) may require adjustment of the chemotherapy given. OXA-NTS have two different manifestations: acute side effects that occur immediately or in hours or days after infusion (e.g. cold-related paraesthesia, dysesthesia), usually resolving within a week, and that have disappeared before the next cycle of chemotherapy; and chronic side effects (e.g. paraesthesia, numbness) leading to functional deficits in the longer term. OXA-NTS can be an intolerable burden and may affect the patient's perceived health-related quality of life ${ }^{4}$.

Findings from a qualitative study with repeated interviews showed that the patients balanced the neurotoxic side effects against their survival. The patients expected to be cured by the cancer treatment and looked forward to a return to life as before the diagnosis, but instead they realised they had to adapt to the remaining neurotoxic side effects. Different strategies were used to manage the neurotoxic side effects and the patients re-prioritised or avoided activities due to the side effects, to handle their daily life ${ }^{5}$. A review of the literature pointed out that CRC patient's experience of distressing symptoms need to be identified to deliver effective symptom management interventions ${ }^{6}$. 
Stringent dose modification is the guidance in clinical praxis to prevent chronic NTS, but few interventions with evidence exist for treatment ${ }^{2,3,7}$. Early identification of OXA-NTS is necessary to enhance the possibility of eliminating or decreasing chronic OXA-NTS and to make successful interventions (e.g. dose reduction), but discrepancies between patients and health care professionals regarding OXA-NTS prevalence exist ${ }^{8,9}$.

Therefore, patient-reported outcomes (PRO) of OXA-NTS during and after treatment are important to provide a comprehensive and accurate assessment of OXA-NTS severity and their impact on the patient's daily activities. These patients treated with OXA have higher survival rate, and commonly used PROs may miss relevant information about OXA-NTS and their effects on daily activities ${ }^{10}$.

This study aimed to identify and assess patient-reported OXA-induced neurotoxic side effects and their impact on the patient's daily activities, during and after chemotherapy.

\section{Theoretical Framework}

The theoretical framework in this study is Orem's self-care deficit nursing theory ${ }^{11}$. Orem introduced patient-related concepts to self-care; self-care agency, which is the capacity of individuals to care for themselves, self-care demand, which involves the actions required to meet a person's self-care needs, and self-care deficit, which occurs when people can no longer meet their self-care needs and require nursing care. Health is seen in relation to self-care deficits. Orem suggests that self-care agency and self-care behavior can be influenced by a wide range of factors, which can include gender, age, environment, family and the healthcare system ${ }^{11}$. The 
ability to balance needs is central, both at the conceptual level and in the care of patients. The nurse's responsibility is to support patients in promoting self-care agency to maintain health. Therefore, nursing is dynamic in order to support patients' needs that may change over time. Preventive health care and primary prevention involves meeting patients' universal self-care requisites effectively. Secondary prevention involves early detection of side effects (interventions), while tertiary prevention occurs when rehabilitation takes place ${ }^{11}$. Hence, communication about patient's neurotoxic side effects is important over time, to individualize self-care advices.

\section{Methods}

\section{Design and participants}

Patients were recruited from four hospitals (two university hospitals and two regional hospitals) in Sweden from September 2013 to June 2016. Inclusion criteria were: patients with CRC, $\geq 18$ years of age, ability to speak and understand Swedish, and treatment with 5-fluorouracil and folinic acid in combination with OXA postoperatively in an adjuvant setting for stage II-III CRC. Exclusion criteria were: patients with reduced cognitive function or recognised earlier neurotoxic side effects. Physicians decided if patients were able to participate in the study. At the initial visit for treatment planning, the patients were given written and oral information about the study by the nurses, who also provided further information and obtained written informed consent. A total of 96 patients were assessed by nurses for eligibility during the recruitment period, and 50 agreed to participate and signed an informed consent form. Due to medical deterioration and changes to the treatment plan, four patients were excluded (Figure 1). All of the remaining 46 patients were 
chemo naïve. The study was performed in accordance with the Declaration of Helsinki and was approved by the Regional Ethical Review Board (record no: 2012/301-31). ClinicalTrials.gov: NCT02412683.

\section{Questionnaire}

To measure the frequency, severity and impact on daily activities of OXA-NTS, a 29-item questionnaire OANQ (the Oxaliplatin-Associated Neurotoxicity Questionnaire) developed by

Leonard et al. was used ${ }^{12}$. The questionnaire is divided into three sections corresponding to the side effect locations: upper extremities (10 items), lower extremities (nine items), and the face/mouth area (10 items). Patients first assessed whether they had the specific side effect, yes or no. If they answered yes, they graded the side effects on a five-point scale ranging from $1=$ "hardly any" to 5= "very much". After that, they graded the impact of OXA-NTS on daily activities, $1=$ "hardly at all bothered" to $5=$ "extremely bothered" in a functional sense ${ }^{12}$. Translation of the OANQ to Swedish and back-translation to English was conducted in three steps. First, a native translator translated all items into Swedish. Secondly, this version was checked by the authors and was then independently back-translated into English by a nativespeaking translator. Thirdly, the original and back-translated versions were compared for equivalence ${ }^{13,14}$. The translation and back-translation of the OANQ were equivalent to the original, and no cultural dilemmas were found. Understanding of items and language was evaluated (five patients, not included in the present study). Tests of validity and reliability were performed and reported separately. Internal consistency was strong for the three domains of the scale $(\alpha>$.840). Test-retest indicated that the OANQ was stable. Intraclass Correlation (ICC) for symptom items and effect on daily activities items showed overall excellent reproducibility. The 
weighted kappa for symptom items and daily activities-items showed overall feasibility ${ }^{15}$.

Driessen et al. validated the questionnaire against FACT/GOG Ntx and they found that both instruments had similar construct validity ${ }^{16}$.

\section{Real-time assessment}

Monitoring side effects with mobile-phone technology have the potential to improve communication between healthcare professionals and patients in cancer care. Patients with cancer discharged from hospital have positive experiences of reporting side effects using mobilephone technology and it is useful throughout the cancer-care trajectory ${ }^{17}$. Assessment of OXANTS was performed in real time, using the Swedish version of the OANQ in a mobile phonebased system (21st Century Mobile AB, http:// www.cqmobil.se) ${ }^{15,17}$, at baseline before the initial OXA treatment cycle (C), the day before (d-1) and two days (d2) after each OXA treatment. The evaluation time-points were selected to capture NTS appearance; the day before (d-1) since there should be no NTS and two days (d2) after since the acute NTS should had disappeared. Follow-up assessments were performed 3, 6, 9 and 12 months after the last OXA treatment. A study procedure was established that included instructions for nurses in using the mobile phone-based system. The patients received oral and written information from the nurses regarding installation of the system on their mobile phones (it was a platform-independent system compatible with JAVA ME phones, iPhone, Android, iPad, and Windows Phones).

When the nurses initiated the transfer of the questionnaire to the individual mobile phone, they used a calendar function to enhance individual customised assessments adapted to each patient's specific OXA chemotherapy regime. The patients had 24 hours to answer the questionnaire, and a reminder to answer was sent at $4 \mathrm{pm}$. The majority of patients used their own mobile phones 
and a few patients used a mobile phone provided by the research team. The patient-reported data were transferred to a secure database and answers were available in real-time, web-based graphs that could be viewed after logging in to a web interface.

\section{Data management and statistics}

Descriptive statistics for the clinical patient characteristics at baseline were performed as means and standard deviations (SD), medians and ranges or proportions, as appropriate. Differences between included and not included patients were tested with a two-sided z-test for proportions, and mean age differences were tested in a two-sided t-test ${ }^{18}$. Longitudinal grading of side effects and impact on daily activities were presented as stacked bar charts showing the distribution in percent. Due to the small sample in time points Cycle 7, day before (d-1) to Cycle 11, two days (d2) after OXA treatment, those were excluded in Figure 2. The sample were initially calculated for 50 patients. The included patients were 50 , but 4 patients were excluded due to medical deterioration and change of treatment plan.

With a dichotomisation of the grading of side effects, the presence or not of neurotoxicity was analysed for each item. Differences among time points were tested with the non-parametric McNemar's test. Since there was a need for a continuous and summarised measurement to perform aggregated longitudinal analysis, a Neurotoxicity Index was calculated by combining the grades for severity of side effects and their effect on daily activities. 
The following formula was developed for this purpose:

$$
\sum_{\text {item } 1}^{\text {item } 29}(\text { side effect grade } *(\text { effect on daily activities grade }+1))
$$

A total of maximum score in side effects (5= "very much") and impact on daily activities (5= “extremely bothered") generated a score of 30 in every item. The Neurotoxicity Index has the potential to measure from 0 up to the highest score of 870 . The index is presented as mean with a $95 \%$ confidence interval (CI) for the total study population and for each sex. Differences among time points Cycle 1, day before (d-1) (baseline), Cycle 5, two days after (d2) (mean of treatment length) and follow-ups after treatment (3, 6, 9 and 12 months) were assessed with the nonparametric Wilcoxon signed rank test. Differences in the Neurotoxicity Index between sexes at each time point were tested with a two-sided t-test, with p-values adjusted with the Bonferroni method.

For the purpose of this longitudinal design with unbalanced data a linear mixed effect model for repeated measures with a first-order autoregressive covariance matrix was fitted. The Neurotoxicity Index is presented as mean with a $95 \%$ CI adjusted for covariates, sex, age and OXA dose as fixed effects and the patients as random effects. The OXA dose entered was the average delivered dose until each specific time point and an interaction term (age*OXA dose) was added to the model due to the correlation between this two variables. The Akaike Information Criterion (AIC) was used to select the final model ${ }^{19}$. Due to the small sample in time points Cycle 7, day before (day -1) to Cycle 11, two days (d2) after OXA treatment, those were excluded in Figure 3. All tests had a significance level of $p<0.05$. All analyses were performed in IBM SPSS Statistics 22. 


\section{Results}

No statistical differences were found in age or sex between the included patients and the patients who declined to participate. The 46 not included patients had an age range of 43-75 years (mean 61, SD 8, median 64) and 29 were male (63\%) (Figure 1). Out of these 46 not included patients, $13(28 \%)$ did not understand the Swedish language. The demographic characteristics of the 46 included patients (provided data for this study) are summarised in Table 1. In total, 370 (297 during treatment, 73 in follow-up) OANQ questionnaire responses were available for analysis (min $1 / \max 26$, mean $8 /$ patient). There was a response rate of $76 \%$ during the OXA treatment (cycle 1-11) and $55 \%$ during the follow-up period, in total $70 \%$. No internal data was missing. At different time points, totally 14 patients withdrew from the study; two died, nine discontinued due to deteriorating health condition, and three had technical/practical problems which could not be resolved (Figure 1). Post treatment, the number of returned questionnaires after three months was $20(56 \%)$, after six months $20(61 \%)$, after nine months $20(61 \%)$ and after 12 months 13 (41\%). Sex distribution in follow-up (male/female): 3 months (55\%/45\%), 6 months (50\%/50 $\%), 9$ months (40\%/60\%) and 12 months (46\%/54\%). The patients received an OXA dose between 75-292 mg (mean $185 \mathrm{mg}$ ) during a total of 227 cycles (1-11 cycles, mean 5) (Table 2). The total dose in the range 120-2428 mg (mean $925 \mathrm{mg}$ ). Dose reduction was applied to all patients at some point.

\section{Oxaliplatin-induced neurotoxic side effects}

All patients in the study reported multifactorial OXA-NTS during OXA-treatment. A majority of the patients reported cold-precipitated tingling, burning pain or discomfort and throat discomfort during OXA treatment. Differences between time points with McNemar's test for each item are 
presented in Table 3. Tingling in the upper extremities was reported by the majority during treatment, and significant differences were found between baseline and the 3-, 9- and 12-month follow-ups. Half of the patients had difficulties perceiving differences between rough and smooth surfaces and significant differences were found between baseline and the 9-month follow-up. One year after treatment, numbness in the lower extremities was significantly different from baseline. Oral/facial NTS were frequently reported during OXA treatment but decreased after the treatment. Some of the face-related side effects such as problems with eyelids or pain in the ears remained relatively unchanged, at a low level, throughout the study period. The results show significant differences between baseline data, and nine of the patients had not returned to baseline after one year. The nine patients who still reported neurotoxicity and effect on daily activities were between 54 and 68 years of age (mean $65, \mathrm{SD} \pm 4$ ). Seven of them were men $(78 \%)$. The four patients who did not report side effects after one year were all females (6169 years, mean 64$)$.

\section{Impact on daily activities}

The patients reported a high frequency of impact on daily activities due to the side effects. In Figure 2, five different items from all three domains are illustrated. Longitudinal grading of side effects and impact on daily activities were presented as stacked bar charts showing the distribution in percentages (dark stacked bars show severe NTS and highly affected daily activities). Figure 2 a-c shows the longitudinal grading of neurotoxic side effects in the upper extremities and the impact on daily activities. Tingling (pins and needles) affected the patient's daily activities over the entire study period (2a). Burning pain or discomfort without cold (2b) were graded by the patients as having a lower impact on daily activities than burning pain or 
discomfort with cold especially during treatment (2c). Tingling (pins and needles) in the lower extremities (2d) affected daily activities more after treatment. Similar results are available regarding numbness in the lower extremities (not shown). In the long-term perspective, side effects in the lower extremities were reported as having a high influence on the patient's daily activities. Oral/facial NTS were frequently reported during OXA treatment, but both neurotoxicity and impact on daily activities decreased after the treatment. Throat discomfort (2e) showed a decreased progression over time.

A Neurotoxicity Index was calculated by combining the grades on severity of side effects and their effect on daily activities. Significant differences were found in the Wilcoxon signed rank test between baseline data of the Neurotoxicity Index and Cycle 5, day 2 (mean value of end of therapy). Significant differences were also found between baseline data and 3-, 6-, 9-, and 12month follow-up time points. No significant differences between the Neurotoxicity Index in Cycle 5, day 2 and the Neurotoxicity Index in the follow-up time points (3, 6, 9 and 12 months) were found $(\mathrm{p}<.05)$. No statistically significant differences were noted between sex and Neurotoxicity Index except for the time point Cycle 6, day $2(\mathrm{p}=.043)$.

Figure 3 show the index throughout the study period. For the purpose of this longitudinal design, a mixed effect model for repeated measures illustrates the patient-reported outcomes of Neurotoxicity Index (adjusted for covariates; age, sex and OXA dose per cycle) during different time points (Figure 3). 


\section{Discussion}

The main result of this study is that OXA-NTS are common and have an impact on daily activities, both during and after OXA treatment. We found significant differences between baseline data and follow-up time points of neurotoxicity, and the patients not returned to baseline after one year. Tingling in the upper extremities was reported by the majority of patients during treatment, and significant differences were found between baseline and follow-up. In the present study, the patients reported that OXA-NTS changed in character and body location over time. The side effects had an impact on the daily activities for the patients, both in the short- and longterm perspectives. NTS was graded as moderate to severe in the lower extremities after completing the OXA treatment.

A previous qualitative study of patients' own statements about OXA-NTS and how they affected their daily life showed that the patients had different strategies to manage the neurotoxic side effects and they re-prioritised or avoided activities due to the side effects in order to handle their daily life. The patients expected to be cured by the cancer treatment, but instead they realised they had to adapt to the remaining neurotoxic side effects and learn to live with the side effects in daily life $^{5}$.

Our results about the prevalence of neurotoxicity in some items (numbness and tingling) one-year post- OXA treatment are in contrast with earlier quantitative studies where OXA-NTS were partly resolved in about $40 \%$ of the patients after six to eight months and were completely resolved one year post-OXA ${ }^{20-22}$. We found significant differences between baseline data and follow-up time points of neurotoxicity. The results also show that the existing neurotoxicity had an impact on daily activities one-year post-OXA. Different measurement methods and study designs in earlier clinical trials make them difficult to compare with our longitudinal study which used real-time 
assessments. A larger number of patients will be required to provide more robust results. Observer reports and retrospective assessments probably show a lower severity of side effects, and coldrelated side effects might be more bothersome in this study population than in studies in warmer countries (Sweden is a country with a cold climate).

At present, actions for managing OXA-NTS are limited ${ }^{23-25}$ and patients often need to learn how to manage the side effects on their own in daily life ${ }^{5,26}$. A study shows that the occurrence of hyperacute OXA-NTS can be a predictor of chronic neurotoxicity, which highlights the importance of careful monitoring ${ }^{27}$. Mobile phone-based systems are useful to monitor the progression of NTS and their impact on daily activities ${ }^{17}$, and patients discharged from hospital have positive experiences of reporting side effects using mobile phone technology ${ }^{28}$. The fact, that this patients had long-term survival and cancer is a chronic disease, future directions, monitoring and management is important. Education and supportive care, may reduce distress and improve perceptions of confidence and control ${ }^{11,29}$.

Monitoring patient's side effects and symptoms will enhance decision making about dose reduction or discontinuation the treatment. Additionally, monitoring of OXA-NTS is important in a long term perspective especially for cancer survivors to eliminate injuries. Real-time patientreported OXA-NTS could be valuable in the clinical setting with continuous individualised monitoring to help identify patients who need further evaluation or healthcare intervention to improve their health. Supportive care is important in this patient group and can be viewed as a patient-centred approach to effectively identifying neurotoxicity and its impact on the patient's daily activities. Nursing support in cancer care includes communication and decision-making and may change over time ${ }^{11}$, and oncology nurses need to use various interventions to provide evidence-based symptom management ${ }^{30}$. 
A strength of this study is the real-time assessments, which seem to be an accurate and valid method for follow-up of PROs. There may be an advantage that the patients in this study were observed and dose-adjusted to a greater extent, which resulted in fewer experienced and reported side effects. Facilitating communication and shared decisions in the clinical practice, may increase quality improvement both in the short and long term. Swedish National Quality Register plan to extend their array of patient reported measures (PROMs) in the future ${ }^{31}$, and PROs about neurotoxicity seem to be relevant to incorporate in the register.

\section{Methodological Considerations}

The sample size of included patients was relatively small. A larger number of patients and a longer follow-up period will be required to provide more robust results ${ }^{18}$. Aiming for larger sample, patient recruitment was extended to four hospitals in a larger geographical area. Ongoing parallel studies recruited the same patient population and due to staff situation at the hospitals no patients were recruited during late spring to early autumn. The sample size in the study may limit the generalizability of the study, but this longitudinal study defines the trajectory of neurotoxicity and impact on daily activities over time.

Patients were included on the basis of the inclusion and exclusion criteria ${ }^{18}$. Selection bias is always a risk with a convenience sample, as used in this study. It should be taken into consideration that there may be a risk that patients who were positive about the mobile phone system, participated to a greater extent. More males were included in the study (61\%), and few patients were included with an educational level lower than high school (11\%). A limitation is the generalization of the results to patients with low literacy level. 
To ensure low dropout rates, a reminder was sent out to the patients' mobile phones. The patients only had one day to answer the questions, and only one reminder was sent out. On the other hand, the response rate was $70 \%$ in total over the period, and the mobile phone-based system ensured that no internal data were missing. There may also be an advantage that the patients in the study were observed and dose-adjusted to a greater extent, which resulted in fewer treatments and also experienced and reported side effects. It is a limitation that only thirteen of the patients responded to the questionnaire after one year. But according to the ethical guidelines, the patients were informed that they could decline to participate or could withdraw from the study at any time without explanation or consequences for their future care.

A limitation to consider, patients withdrew from the study at different time points. For the purpose of this longitudinal design with unbalanced data a linear mixed effect model for repeated measures was used. In McNemar's test (Table 3), only matched pairs of data was analysed and presented. Due to the small sample in time points Cycle 7, day before (day -1) to Cycle 11, two days (d2) after OXA treatment, those were excluded in Figure 2 and 3.

Confounding factors such as alcohol consumption, smoking habits, e.g. vitamin $\mathrm{B}_{12}$ deficiency or other diseases may have changed over the follow-up-period, which may have affected longitudinal outcomes. Another confounder we did not study was the patient's body weight and body mass index (BMI). Obesity and BMI over 30 may increase the risk of neurotoxic side effects ${ }^{32}$. 


\section{Implications for Practice}

Real-time patient-reported OXA-NTS could be valuable in the clinical setting with continuous individualised monitoring to help identify patients who need further evaluation or healthcare intervention to improve their health. Supportive care is important in this patient group and can be viewed as a patient-centred approach to effectively identifying neurotoxicity and its impact on the patient's daily activities. Monitoring OXA-NTS is important both in the short (e.g. dosereduction) and long term (e.g. rehabilitation). The results could be used to further develop healthcare interventions. Patient reported outcomes should be the basis for tailored care for these patients.

\section{Conclusions}

In conclusion, the high prevalence of OXA-NTS significantly interferes with the patient's daily activities in different ways, both in the short and long term. We found significant differences between baseline data and follow-up time points in regard to neurotoxicity, and all of the patients had not returned to baseline after one year. The results suggest the need for tailored care for these patients, to reduce or eliminate chronic NTS in the long term. Our results stresses the need of early detection and also monitoring both in the short and long term perspective. Mobile-phone technology enhance patient's participation in their own self-care. These results should be taken into account when patients are informed about the benefits and also the risks of adjuvant OXA treatment. 


\section{References}

1. Okamoto I, Wright D, Foster C. Impact of cancer on everyday life: a systematic appraisal of the research evidence. Health Expect. 2012;15:97-111.

2. André T, Boni C, Navarro M, et al. Improved overall survival with oxaliplatin, fluorouracil, and leucovorin as adjuvant treatment in stage II and III colon cancer in the MOSAIC trial. J Clin Oncol 2009;27:3109-3116.

3. Park S, Goldstein D, Krishnan A et al. Chemotherapy-Induced Peripheral Neurotoxicity: A Critical Analysis. CA Cancer J Clin 2013;63:419-437.

4. Stefansson M, Nygren P. Oxaliplatin added to fluoropyrimidine for adjuvant treatment of colorectal cancer is associated with long-term impairment of peripheral nerve sensory function and quality of life. Acta Oncol 2016;55:1227-1235.

5. Drott J, Starkhammar H, Kjellgren K et al. The trajectory of neurotoxic side effects' impact on daily life: a qualitative study. Support Care Cancer 2016;24:3455-3461.

6. Tantoy IY, Cataldo JK, Aouizerat BE et al. A review of the literature on multiple co-occurring symptoms in patients with colorectal cancer who received chemotherapy alone or chemotherapy with targeted therapies. Cancer Nursing 2016;39(6):437-445.

7. Brewer J, Morrison G, Dolan E et al. Chemotherapy-induced peripheral neuropathy: Current status and progress. Gynecologic Oncol 2016;140:176-183.

8. Cirillo M, Venturini M, Ciccarelli L et al. Clinician versus nurse symptom reporting using the National Cancer Institute-Common Terminology Criteria for Adverse Events during chemotherapy: results of comparison based on patient's self-reported questionnaire. Ann Oncol 2009;20:1929-1935. 
9. Alberti P, Rossi E, Cornblath D.R et al. Physician-assessed and patient-reported outcome measures in chemotherapy-induced sensory peripheral neurotoxicity: two sides of the same coin. Ann Oncol 2014; 25:257-264.

10. Catt S, Starkings R, Shilling V et al. Patient-reported outcome measures of the impact of cancer on patients' everyday lives: a systematic review. J Cancer Surviv 2017;11: 211-232.

11. Orem D. Nursing: concepts of practice. Mosby; 2001: St. Louis.

12. Leonard GD, Wright MA, Quinn MG et al. Survey of oxaliplatin-associated neurotoxicity using an interview-based questionnaire in patients with metastatic colorectal cancer. BMC Cancer $2005 ; 16: 5: 116$.

13. Acquadro C, Conway K, Giroudet $\mathrm{C}$ et al. Linguistic validation manual for health outcome assessments. MAPI Institute; 2012: Lyon.

14. Brislin RW, Back-translation for cross-cultural research. J Cross-Cultur Psychol. 1970; 1:185216.

15. Gustafsson E, Litström E, Berterö C, et al. Reliability testing of oxaliplatin-associated neurotoxicity questionnaire (OANQ), a pilot study. Support Care Cancer 2016;24:747-754.

16. Driessen C, de Kleine-Bolt KM, Vingerhoets AJ et al. Assessing the impact of chemotherapyinduced peripheral neurotoxicity on the quality of life of cancer patients: the introduction of a new measure. Support Care Cancer 2012;4:877-81.

17. Drott J, Vilhelmsson M, Kjellgren K et al. Experiences with a self-reported mobile phone-based system among patients with colorectal cancer: A qualitative study. JMIR Mhealth Uhealth 2016; 4:e66.

18. Polit D.F, Beck C.T. Nursing research: generating and assessing evidence for nursing practice. Wolters Kluwer, Philadelphia; 2016. 
19. Brown H, Prescott R. Applied Mixed Models in Medicine. John Wiley \& Sons; 2015.

20. Argyriou A. Kyritsis A, Makatsoris T, et al. Chemotherapy-induced peripheral neuropathy in adults: a comprehensive update of the literature. Cancer Manag Research 2014;6:135-147.

21. Beijers A, Mols F, Dercksen W, et al. Chemotherapy-induced peripheral neuropathy and impact on quality of life 6 months after treatment with chemotherapy. J Community Support Oncol. 2014;12(11):401-6.

22. Argyriou AA, Zolota V, Kyriakopoulou O, et al. Toxic peripheral neuropathy associated with commonly used chemotherapeutic agents. J Buon 2010;15(3):435-446.

23. Fox P, Darley A, Furlong E, et al. The assessment and management of chemotherapy-related toxicities in patients with breast cancer, colorectal cancer, and Hodgkin's and non-Hodgkin's lymphomas: A scoping review. Eur J Oncol Nurs 2017;26:63-82.

24. Hershman DL, Lacchetti C, Dworkin RH, et al. Prevention and management of chemotherapyinduced peripheral neuropathy in survivors of adult cancers: American Society of Clinical Oncology clinical practice guideline. J Clin Oncol. 2014;32(18):1941-1967.

25. Majithia N, Temkin SM, Ruddy KJ, et al. National Cancer Institute-supported chemotherapyinduced peripheral neuropathy trials: outcomes and lessons. Support Care Cancer. 2016;24(3):1439-1447.

26. Tanay MA, Armes J, Ream E. The experience of chemotherapy-induced peripheral neuropathy in adult cancer patients: a qualitative thematic synthesis. Eur J Cancer Care. 2016;Jan 20: doi: 10.1111/ecc. 12443 .

27. Tanishima H, Tominaga T, Kimura M, et al. Hyperacute peripheral neuropathy is a predictor of oxaliplatin-induced persistent peripheral neuropathy. Support Care Cancer 2017;25(5):13831389. 
28. Mirkovic J, Kaufman D.R, Ruland C.M. Supporting cancer patients in illness management: usability evaluation of a mobile app. JMIR Mhealth Uhealth 2014;2(3):e33.

29. Hammer M, Ercolano E, Wright F, et al. Self-management for adult patients with cancer. Cancer Nursing 2015;38(2):E10-E26.

30. Henoch I, Olsson C, Larsson M, et al. Symptom dimensions as outcomes in interventions for patients with cancer: a systematic review. Oncology Nursing Forum 2018;45(2):237-249.

31. Nilsson E, Orwelius L, Kristenson M. Patient-reported outcomes in the Swedish National Quality Registers. Journal of Internal Medicine 2016;279(2):141-153.

32. Cox-Martin E, Trahan L.H, Cox M.G et al. Disease burden and pain in obese cancer patients with chemotherapy-induced peripheral neuropathy. Support Care Cancer 2017;25(6):1873-1879. 


\section{Labels Tables/Figure}

Table 1. Baseline patient and clinical characteristics $(n=46)$

Table 2. Dose of oxaliplatin, mg/cycle $(n=46)$

Table 3. Patient-reported neurotoxicity differences between time-points for each item.

Figure 1. Flow diagram of study enrolment and number of patients at different time points.

Figure 2. Patient-reported neurotoxic side effects and impact on daily activities from five selected OANQ items in three body localisations. Graded from 0="No side effect" to 5= "Very much" and impact on daily activities $0="$ No affect" to 5= "Extremely bothered". Time axis; cycle number (C No.), day before (d-1) and 2 days (d2) after OXA treatment.

Figure 3. Overall longitudinal measure of patient-reported outcomes using the Neurotoxicity Index from the linear mixed effect model (adjusted for covariates; age, sex and OXA dose per cycle) throughout the study period. 


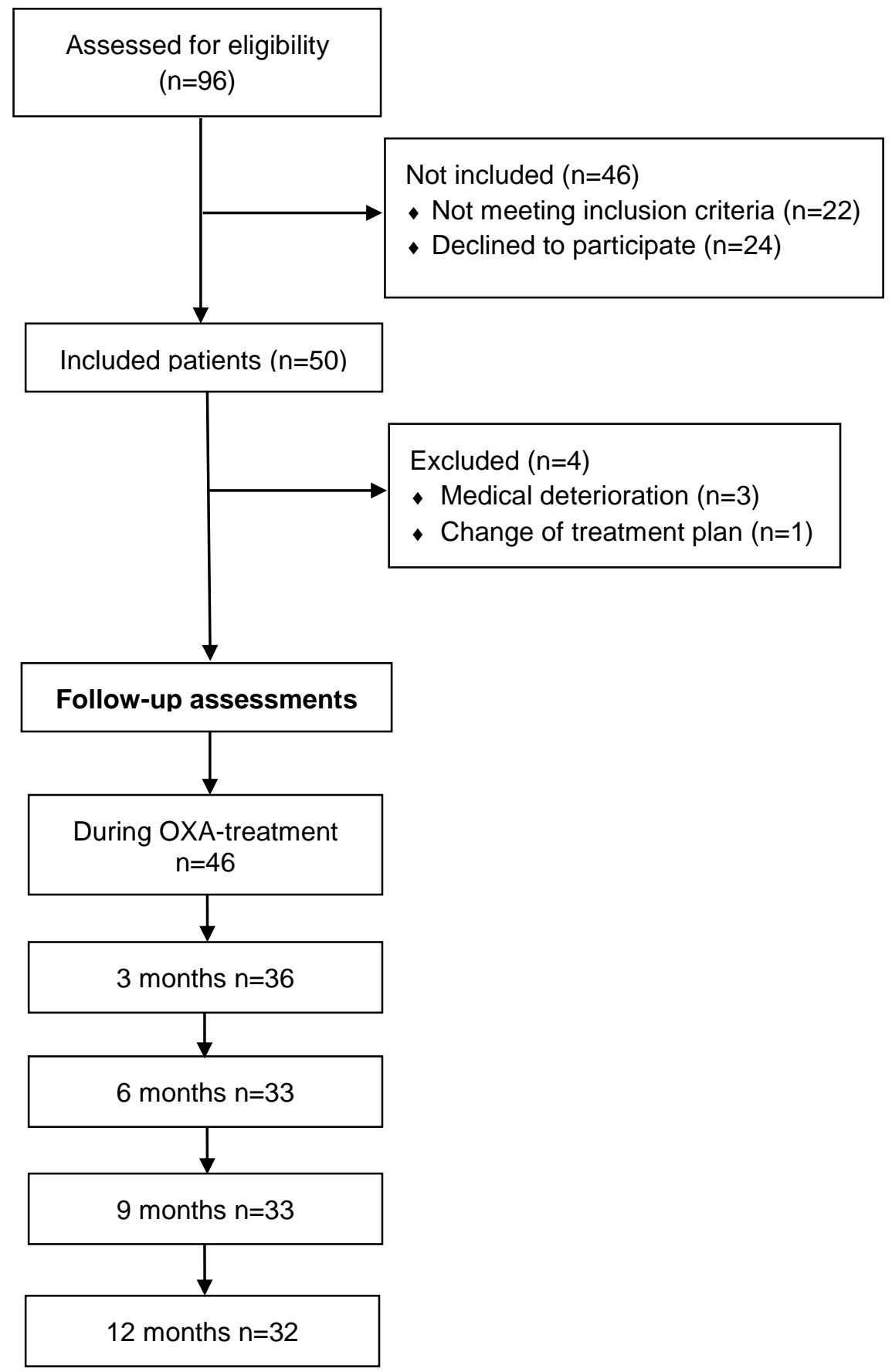

Figure 1. Flow diagram of study enrollment and number of patients at different time points. 
a. Upper extremity: Tingling (Pins and needles)

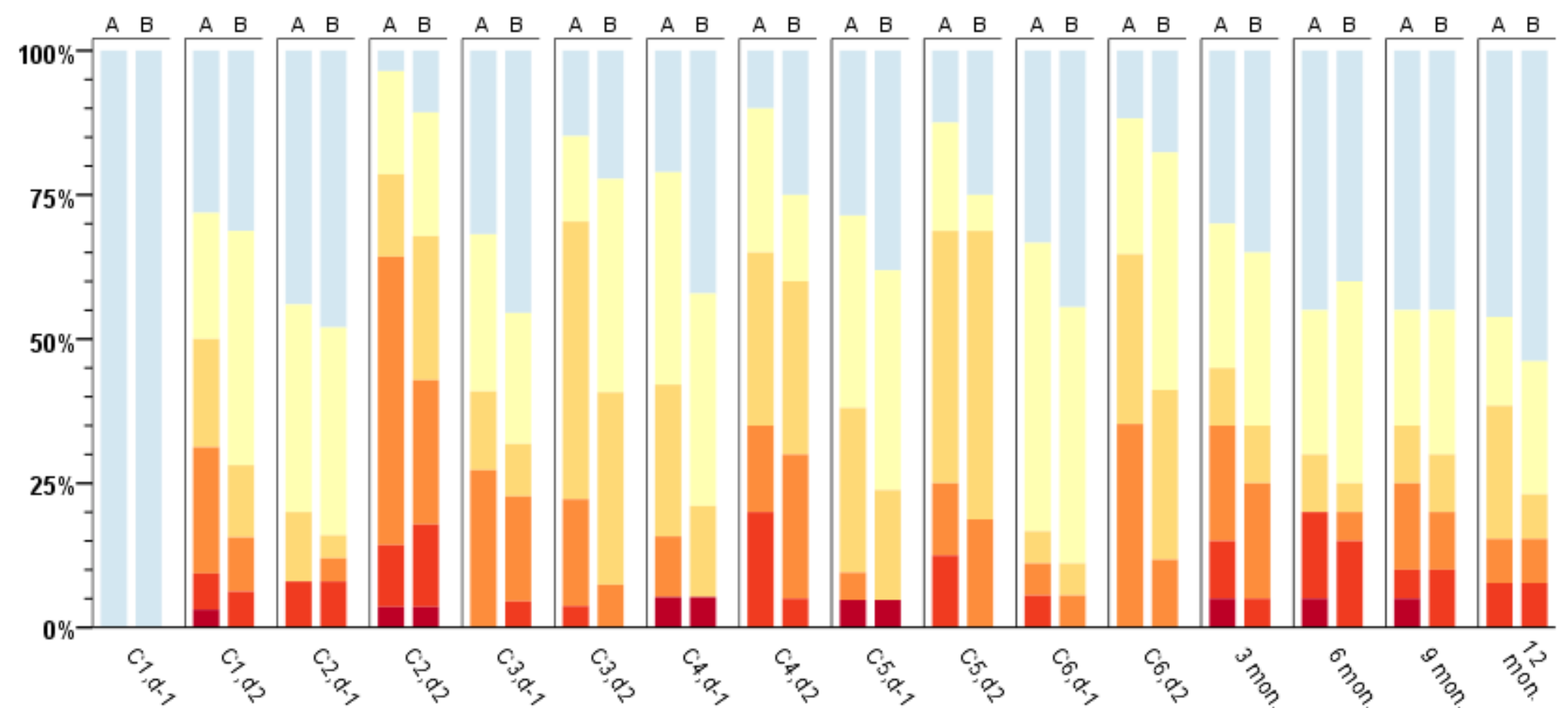

Figure 2. Patient-reported neurotoxic side effects and impact on daily activities from five selected OANQ items in three body localisations. Graded from $0=$ "No side effect" to $5=$ "Very much" and impact on daily activities $0=$ "No affect" to $5=$ "Extremely bothered".

Time axis; cycle number ( $C$ No.), day before ( $d-1$ ) and 2 days (d2) after OXA treatment. 


\section{b. Upper extremity: Burning pain or discomfort without cold}

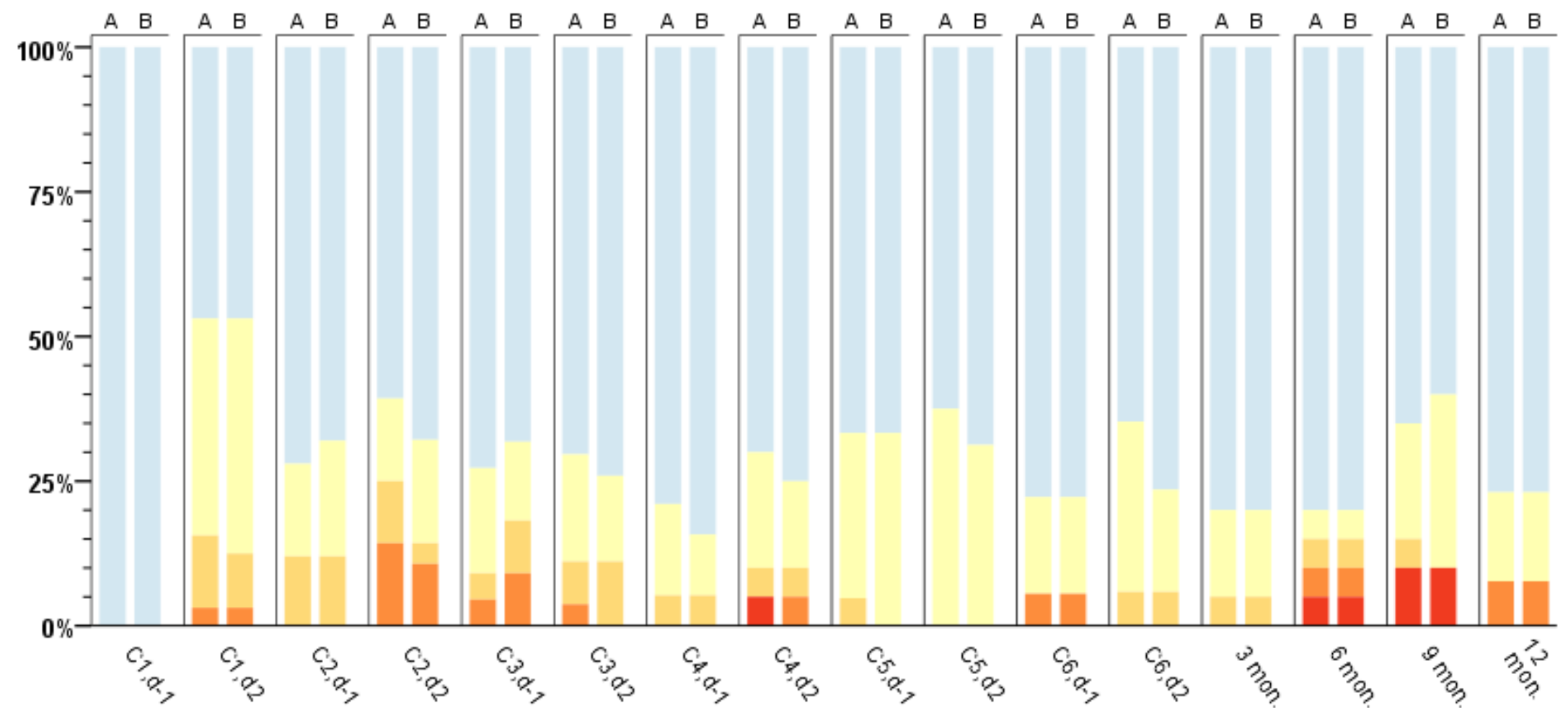

Figure 2. Patient-reported neurotoxic side effects and impact on daily activities from five selected OANQ items in three body localisations. Graded from $0=$ "No side effect" to $5=$ "Very much" and impact on daily activities $0=$ "No affect" to $5=$ "Extremely bothered". Time axis; cycle number (C No.), day before (d-1) and 2 days (d2) after OXA treatment. 


\section{c. Upper extremity: Burning pain or discomfort with cold}

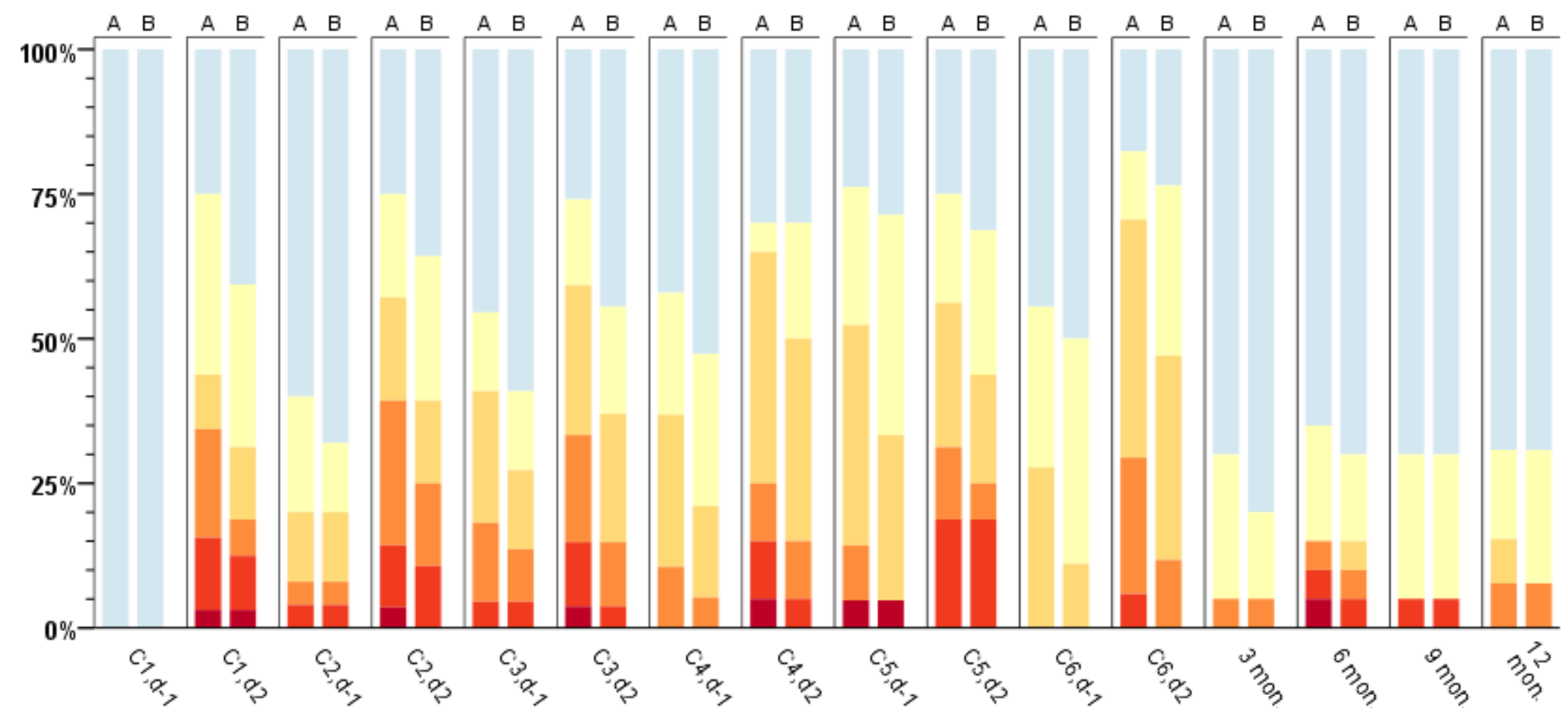

Figure 2. Patient-reported neurotoxic side effects and impact on daily activities from five selected OANQ items in three body localisations. Graded from $0=$ "No side effect" to $5=$ "Very much" and impact on daily activities $0=$ "No affect" to $5=$ "Extremely bothered". Time axis; cycle number (C No.), day before (d-1) and 2 days (d2) after OXA treatment. 


\section{d. Lower extremity: Tingling (pins and needles)}

$A=$ Grade of side effect

$B=$ Affect on daily activities

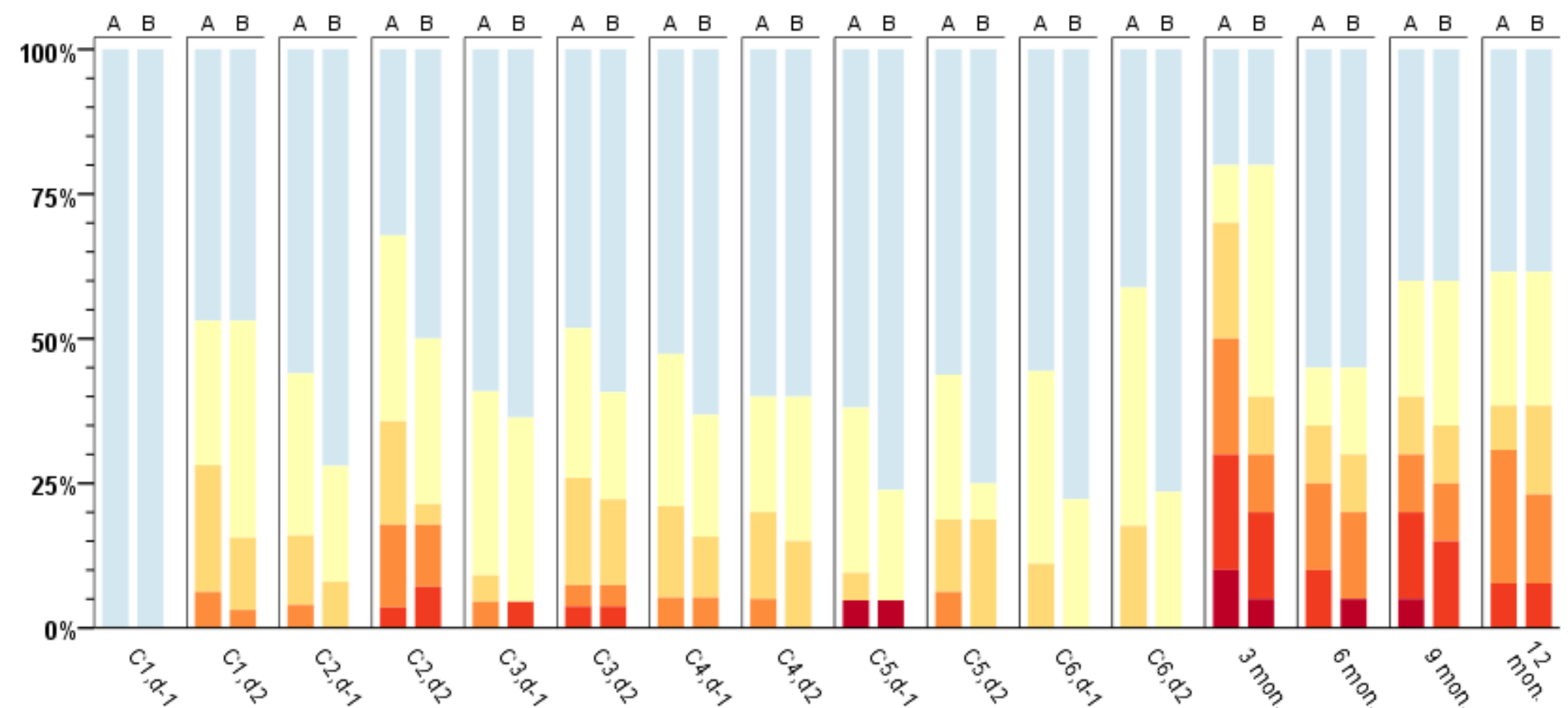

Figure 2. Patient-reported neurotoxic side effects and impact on daily activities from five selected OANQ items in three body localisations. Graded from $0=$ "No side effect" to $5=$ "Very much" and impact on daily activities $0=$ "No affect" to $5=$ "Extremely bothered".

Time axis; cycle number (C No.), day before (d-1) and 2 days (d2) after OXA treatment. 


\section{e. Throat discomfort}

$A=$ Grade of side effect

$B=$ Affect on daily activities

0
1

는

4
5

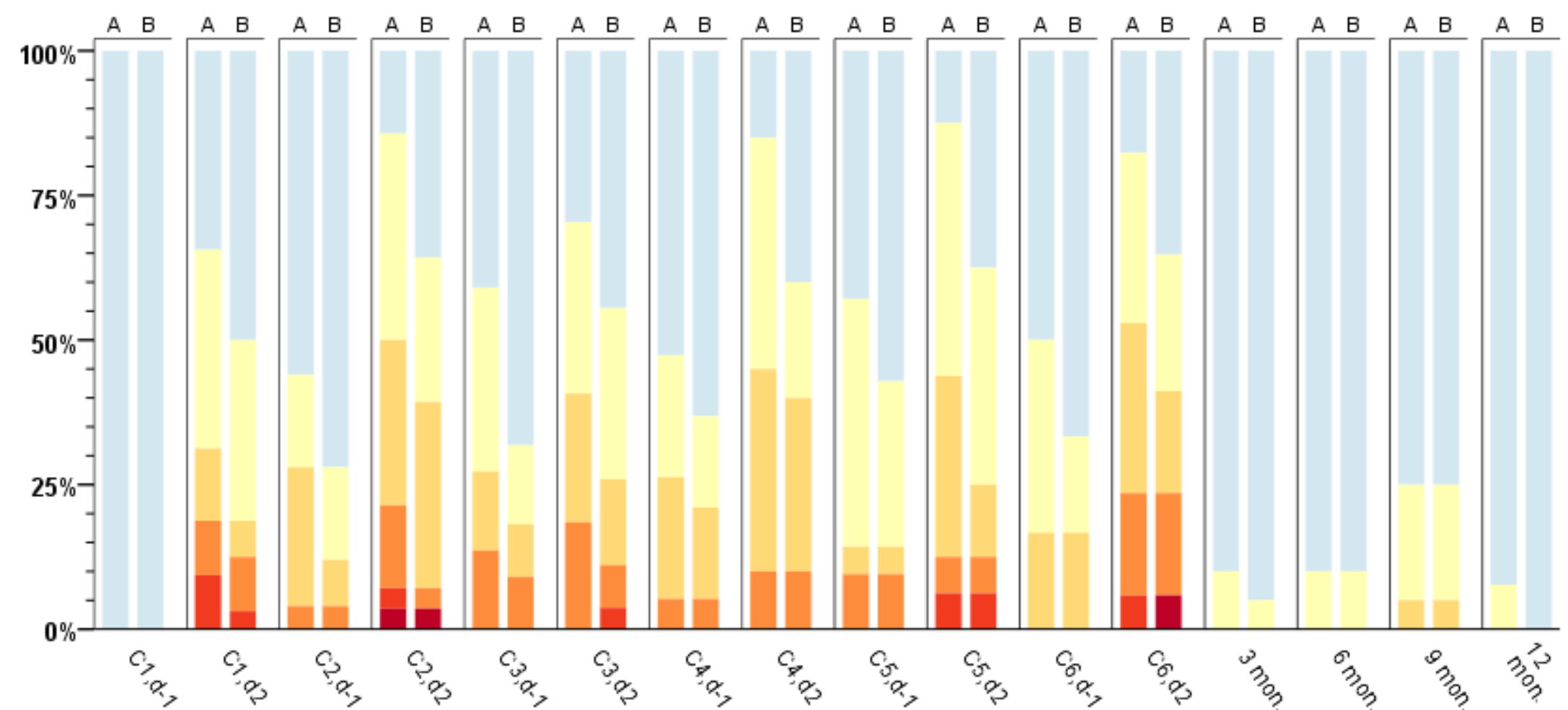

Figure 2. Patient-reported neurotoxic side effects and impact on daily activities from five selected OANQ items in three body localisations. Graded from $0=$ "No side effect" to $5=$ "Very much" and impact on daily activities $0=$ "No affect" to $5=$ "Extremely bothered".

Time axis; cycle number (C No.), day before (d-1) and 2 days (d2) after OXA treatment. 


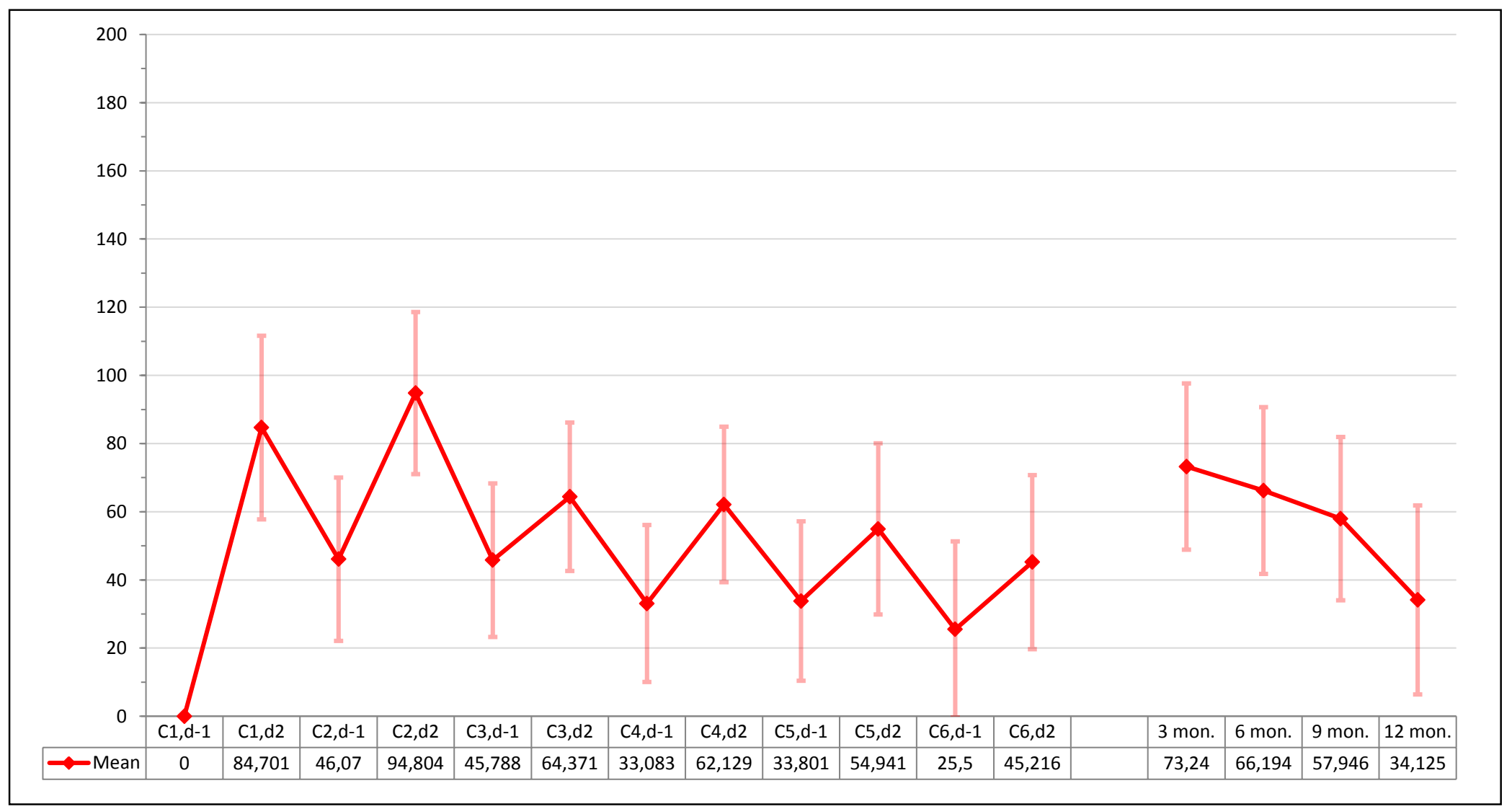

Figure 3. Overall longitudinal measure of patient-reported outcomes using the Neurotoxicity Index from the linear mixed effect model (adjusted for covariates; age, gender and OXA dose per cycle) throughout the study period. 
Table 1 Baseline Patient and Clinical Characteristics $(n=46)$

Age (years) mean (SD), median (range)

Gender, $n(\%)$

Male

Female

Social status, $n(\%)$

Living alone

Living with partner

Living with partner and children at home,

$<21$ years

Education level, $\boldsymbol{n}(\%)$

Less than high school

High school or college

University

$6(13)$

$33(72)$

7 (15)

Occupation, $\boldsymbol{n}(\%)$

Employed full time

Employed part time

Retired

Early retired/Disability

Sick leave full time

Unemployed

Employed part time and sick leave part time

No answer

Type of malignancy, $\boldsymbol{n}(\%)$

Colon

$40(87)$

Rectum

Chemotherapy, $n$ (\%)

FOLFOX, FLOX ${ }^{a}$

XELOX $^{b}$

$39(85)$

Oxaliplatin $\mathrm{mg} / \mathrm{m}^{2}$ (total dose) mean, range

$925,120-2428$

\footnotetext{
${ }^{\text {a }}$ Fluorouracil + leucovorin + oxaliplatin
}

${ }^{\mathrm{b}}$ Capecitabine + oxaliplatin 
Table 2. Dose of Oxaliplatin, $\mathrm{mg} /$ cycle ( $\mathrm{n}=46 ; 7$ patients schedule for FOLFOX; 39 patients for CAPOX). FOLFOX (Oxalipatin 85mg/m²), every two weeks (q2w), planned 12 cycles, CAPOX (Oxaliplatin 130mg/m²), (q3w), planned 8 cycles.

\begin{tabular}{|c|c|c|c|c|c|}
\hline \multicolumn{6}{|c|}{ Total dose per cycle of Oxaliplatin, mg/cycle } \\
\hline Cycle & Mean & $\begin{array}{l}\text { Standard } \\
\text { Deviation }\end{array}$ & Minimum & Maximum & No of patients \\
\hline 1 & 214 & 54 & 120 & 292 & 46 \\
\hline 2 & 204 & 55 & 110 & 292 & 39 \\
\hline 3 & 182 & 61 & 90 & 292 & 37 \\
\hline 4 & 178 & 59 & 90 & 292 & 32 \\
\hline 5 & 175 & 51 & 90 & 270 & 28 \\
\hline 6 & 169 & 55 & 80 & 270 & 25 \\
\hline 7 & 159 & 43 & 90 & 240 & 9 \\
\hline 8 & 132 & 46 & 75 & 170 & 5 \\
\hline 9 & 97 & 26 & 75 & 125 & 3 \\
\hline 10 & 93 & 25 & 75 & 110 & 2 \\
\hline 11 & 75 & 0 & 75 & 75 & 1 \\
\hline 12 & 0 & 0 & 0 & 0 & 0 \\
\hline
\end{tabular}


Table 3. Patient-reported Neurotoxicity Differences Between Time-points for Each Item.

\begin{tabular}{|c|c|c|c|c|c|c|c|c|c|c|c|c|c|c|c|}
\hline & C1. d-1 (Baseline) & \multicolumn{2}{|c|}{ C5. $\mathrm{d} 2$} & \multicolumn{3}{|c|}{3 months follow-up } & \multicolumn{3}{|c|}{6 months follow-up } & \multicolumn{3}{|c|}{9 months follow-up } & \multicolumn{3}{|c|}{12 months follow-up } \\
\hline & $\%$ & $\%$ & $\begin{array}{c}P_{\text {Base- }} \\
\text { C5.d2 }\end{array}$ & $\%$ & $\begin{array}{c}P_{\text {Base- }} \\
\text { 3mon }\end{array}$ & $\begin{array}{c}P_{\text {c5.d2- }} \\
\text { 3mon }\end{array}$ & $\%$ & $\begin{array}{l}P_{\text {Base- }} \\
\text { mmon }\end{array}$ & $\begin{array}{c}P_{c 5 . d 2-} \\
6 \text { mon }\end{array}$ & $\%$ & $\begin{array}{c}P_{\text {Base- }} \\
\text { 9mon }\end{array}$ & $\begin{array}{c}P_{C 5 . d 2-} \\
9 \text { mon }\end{array}$ & $\%$ & $\begin{array}{l}P_{\text {Base- }} \\
12 m o n\end{array}$ & $\begin{array}{l}P_{c 5 . d 2-} \\
12 \text { mon }\end{array}$ \\
\hline \multicolumn{16}{|l|}{ Upper Extremity } \\
\hline Tingling (Pins and needles) & 0 & 88 & .004 & 70 & .016 & .500 & 55 & .063 & 1.000 & 55 & .016 & .625 & 54 & .031 & 1.000 \\
\hline Numbness & 0 & 50 & .063 & 65 & .031 & .063 & 35 & .125 & 1.000 & 45 & .063 & 1.000 & 38 & .125 & 1.000 \\
\hline Difficulty telling the difference between rough and smooth surfaces & 0 & 38 & .250 & 55 & .031 & .219 & 35 & .250 & 1.000 & 55 & .016 & .687 & 31 & .250 & 1.000 \\
\hline Difficulty feeling hot things & 0 & 19 & 1.000 & 35 & .063 & 125 & 25 & 250 & 1.000 & 45 & .031 & .250 & 38 & .125 & 1.000 \\
\hline Difficulty feeling cold things & 0 & 56 & 125 & 40 & .031 & 1.000 & 40 & .250 & .625 & 45 & .031 & .687 & 38 & .125 & .250 \\
\hline A greater than normal sense of touch (i.e.. putting on gloves) & 0 & 44 & .063 & 30 & .125 & .500 & 20 & .500 & .500 & 45 & .031 & 1.000 & 31 & .250 & 1.000 \\
\hline Burning pain or discomfort without cold & 0 & 38 & 125 & 20 & .500 & 1.000 & 20 & .500 & .500 & 35 & .125 & .625 & 23 & .500 & 1.000 \\
\hline Burning pain or discomfort with cold & 0 & 75 & .008 & 30 & .250 & .500 & 35 & .250 & .125 & 30 & .125 & .063 & 31 & .250 & .250 \\
\hline Difficulty identifying objects in your hand (i.e.. coin) & 0 & 19 & 1.000 & 30 & .500 & .125 & 25 & .500 & 1.000 & 35 & .063 & .500 & 23 & .500 & 1.000 \\
\hline Involuntary hand movements & 0 & 56 & .031 & 25 & .500 & 1.000 & 25 & .500 & .125 & 30 & .250 & .625 & 8 & 1.000 & 1.000 \\
\hline \multicolumn{16}{|l|}{$\%=$ percentage with neurotoxicity. $P=p$-value. } \\
\hline & C1. d-1 (Baseline) & \multicolumn{2}{|c|}{ C5. d2 } & \multicolumn{3}{|c|}{3 months follow-up } & \multicolumn{3}{|c|}{6 months follow-up } & \multicolumn{3}{|c|}{9 months follow-up } & \multicolumn{3}{|c|}{12 months follow-up } \\
\hline & $\%$ & $\%$ & $\begin{array}{l}P_{\text {Base- }} \\
\text { C5.d2 }\end{array}$ & $\%$ & $\begin{array}{c}P_{\text {Base- }} \\
\text { 3mon } \\
\end{array}$ & $\begin{array}{c}P_{C 5 . d 2-} \\
\text { 3mon }\end{array}$ & $\%$ & $\begin{array}{l}P_{\text {Base- }} \\
6 \text { mon }\end{array}$ & $\begin{array}{c}P_{\text {C5.d2- }} \\
6 \text { mon }\end{array}$ & $\%$ & $\begin{array}{c}P_{\text {Base- }} \\
\text { 9mon }\end{array}$ & $\begin{array}{l}P_{\text {C5.d2- }} \\
9 \text { mon }\end{array}$ & $\%$ & $\begin{array}{l}P_{\text {Base- }} \\
12 m o n\end{array}$ & $\begin{array}{l}P_{C 5 . d 2-} \\
12 \text { mon } \\
\end{array}$ \\
\hline \multicolumn{16}{|l|}{ Lower Extremity } \\
\hline Tingling (pins and needles) & 0 & 44 & .063 & 80 & .008 & .031 & 45 & .125 & .625 & 60 & .016 & .063 & 62 & .063 & .500 \\
\hline Numbness & 0 & 44 & 125 & 80 & .008 & 125 & 55 & .063 & .250 & 60 & .031 & .375 & 69 & .031 & .500 \\
\hline Difficulty telling the difference between rough and smooth surfaces & 0 & 19 & 1.000 & 45 & .063 & .031 & 40 & .125 & .125 & 55 & .016 & .031 & 46 & .250 & .250 \\
\hline Difficulty feeling hot things & 0 & 19 & 1.000 & 35 & .125 & .250 & 25 & .250 & 1.000 & 50 & .031 & .125 & 38 & .500 & .500 \\
\hline Difficulty feeling cold things & 0 & 44 & .125 & 35 & .250 & .500 & 30 & .250 & 1.000 & 45 & .031 & 1.000 & 46 & .250 & 1.000 \\
\hline A greater than normal sense of touch (i.e.. discomfort with socks) & 0 & 31 & .250 & 55 & .031 & .031 & 40 & .250 & .500 & 50 & .016 & .250 & 46 & .125 & 1.000 \\
\hline Burning pain or discomfort without cold & 0 & 25 & .500 & 40 & .125 & .063 & 25 & .250 & 1.000 & 40 & .031 & .500 & 46 & .125 & .500 \\
\hline Burning pain or discomfort with cold & 0 & 56 & .031 & 30 & .250 & 1.000 & 35 & .125 & .500 & 45 & .016 & 1.000 & 46 & .125 & 1.000 \\
\hline Legs feel heavy & 0 & 69 & .031 & 50 & .125 & 1.000 & 55 & .063 & 1.000 & 50 & .063 & 1.000 & 31 & .250 & .500 \\
\hline
\end{tabular}


Table 3. Patient-reported Neurotoxicity Differences Between Time-points for Each Item.

\begin{tabular}{|c|c|c|c|c|c|c|c|c|c|c|c|c|c|c|c|}
\hline & C1. d-1 (Baseline) & \multicolumn{2}{|c|}{ C5. d2 } & \multicolumn{3}{|c|}{3 months follow-up } & \multicolumn{3}{|c|}{6 months follow-up } & \multicolumn{3}{|c|}{9 months follow-up } & \multicolumn{3}{|c|}{12 months follow-up } \\
\hline & $\%$ & $\%$ & $\begin{array}{l}P_{\text {Base- }} \\
\text { C5.d2 }\end{array}$ & $\%$ & $\begin{array}{c}P_{\text {Base- }} \\
\text { 3mon }\end{array}$ & $\begin{array}{c}P_{\text {C5.d2- }} \\
\text { 3mon }\end{array}$ & $\%$ & $\begin{array}{c}P_{\text {Base- }} \\
\text { 6mon }\end{array}$ & $\begin{array}{c}P_{C 5 . d 2-} \\
6 \text { mon } \\
\end{array}$ & $\%$ & $\begin{array}{c}P_{\text {Base- }} \\
\text { 9mon }\end{array}$ & $\begin{array}{c}P_{C 5 . d 2-} \\
9 \text { mon }\end{array}$ & $\%$ & $\begin{array}{l}P_{\text {Base- }} \\
12 \text { mon } \\
\end{array}$ & $\begin{array}{c}P_{C 5 . d 2-} \\
12 m o n \\
\end{array}$ \\
\hline \multicolumn{16}{|l|}{ Facial/Oral } \\
\hline Jaw pain & 0 & 69 & .016 & 5 & 1.000 & .125 & 10 & 1.000 & .063 & 20 & .500 & .031 & 8 & 1.000 & .500 \\
\hline Eyelids drooping & 0 & 38 & .125 & 15 & 1.000 & 1.000 & 20 & .500 & .500 & 25 & .500 & .500 & 0 & & 1.000 \\
\hline Throat discomfort & 0 & 88 & .004 & 10 & 1.000 & .031 & 10 & 1.000 & .016 & 25 & .500 & .008 & 8 & 1.000 & .125 \\
\hline Ear pain & 0 & 25 & .500 & 20 & .500 & 1.000 & 10 & 1.000 & 1.000 & 15 & 1.000 & .500 & 0 & . & 1.000 \\
\hline Tingling in mouth & 0 & 75 & .016 & 20 & .500 & .031 & 15 & 1.000 & .016 & 15 & 1.000 & .008 & 8 & 1.000 & .250 \\
\hline Difficulty with speech & 0 & 56 & .031 & 15 & .500 & .250 & 10 & 1.000 & .125 & 20 & 1.000 & .031 & 8 & 1.000 & 1.000 \\
\hline Burning or discomfort with your eyes & 0 & 44 & .125 & 15 & .500 & 1.000 & 20 & 1.000 & .125 & 15 & 1.000 & .125 & 15 & 1.000 & 1.000 \\
\hline Loss of any vision & 0 & 56 & .063 & 30 & .250 & 1.000 & 30 & .250 & 1.000 & 20 & .500 & .375 & 8 & 1.000 & .500 \\
\hline Feeling shock/pain down back & 0 & 31 & .500 & 20 & 1.000 & 1.000 & 30 & .500 & 1.000 & 20 & 1.000 & 1.000 & 31 & .500 & 1.000 \\
\hline Problems with breathing & 0 & 38 & .250 & 15 & .500 & .500 & 15 & .500 & 1.000 & 15 & 1.000 & .250 & 15 & 1.000 & 1.000 \\
\hline
\end{tabular}

$\%=$ percentage with neurotoxicity. $P=p$-value.

Abbreviations: Time-points; C1. d-1 $=$ Cycle 1. day before OXA-treatment, C5. d2= Cycle 5. 2 days after OXA-treatment, Base= Baseline,

$\%=$ percentage of patients with neurotoxicity, $P=$ p-value. Bold $=$ statistically significant $(P<.05)$. 